\title{
Techniques for collecting blood from the domestic chicken
}

\author{
Lisa M. Kelly, BS, RLAT \& Leanne C. Alworth, DVM, MS, DACLAM
}

As the use of chickens in biomedical research is increasing, demand is growing for technical skills involving poultry, particularly techniques such as venipuncture. Phlebotomy (blood collection) is an important diagnostic tool for determining causes of morbidity and mortality and for collection of other researchrelevant data. This column describes four standard methods of blood collection from the domestic chicken.

Poultry (Galliformes) are becoming increasing popular as laboratory animals; their biomedical use is quickly joining their traditional role in agricultural research. According to the US National Institutes of Health, the chicken is the "premier non-mammalian research model organism” (http://www.nih.gov/ science/models/gallus/). The domestic chicken (Gallus gallus) has long been important in vaccine development and in the study of metabolism ${ }^{1}$. Chickens also have been used as animal models in the study of atherosclerosis, hypertension and cholesterol management and have been instrumental to discoveries in development, virology, immunology, oncology, genetics and evolution ${ }^{1}$. Some strains spontaneously develop muscular dystrophy, and many are susceptible to viruses that lead to tumor formation, making the chicken a great model for the study of certain cancers ${ }^{1}$. The chicken genome has been sequenced, a large number of genomic resources are available for research, and transgenic chickens have been generated (http://www.nih.gov/ science/models/gallus/). Additionally, the avian egg is very accessible and easily allows for study of embryonic development ${ }^{1}$. There are also multiple practical advantages of using chickens as research models. They are inexpensive to purchase and maintain. If purchased from reputable breeders, their genetic background and health history is commonly documented ${ }^{1}$. In addition, they

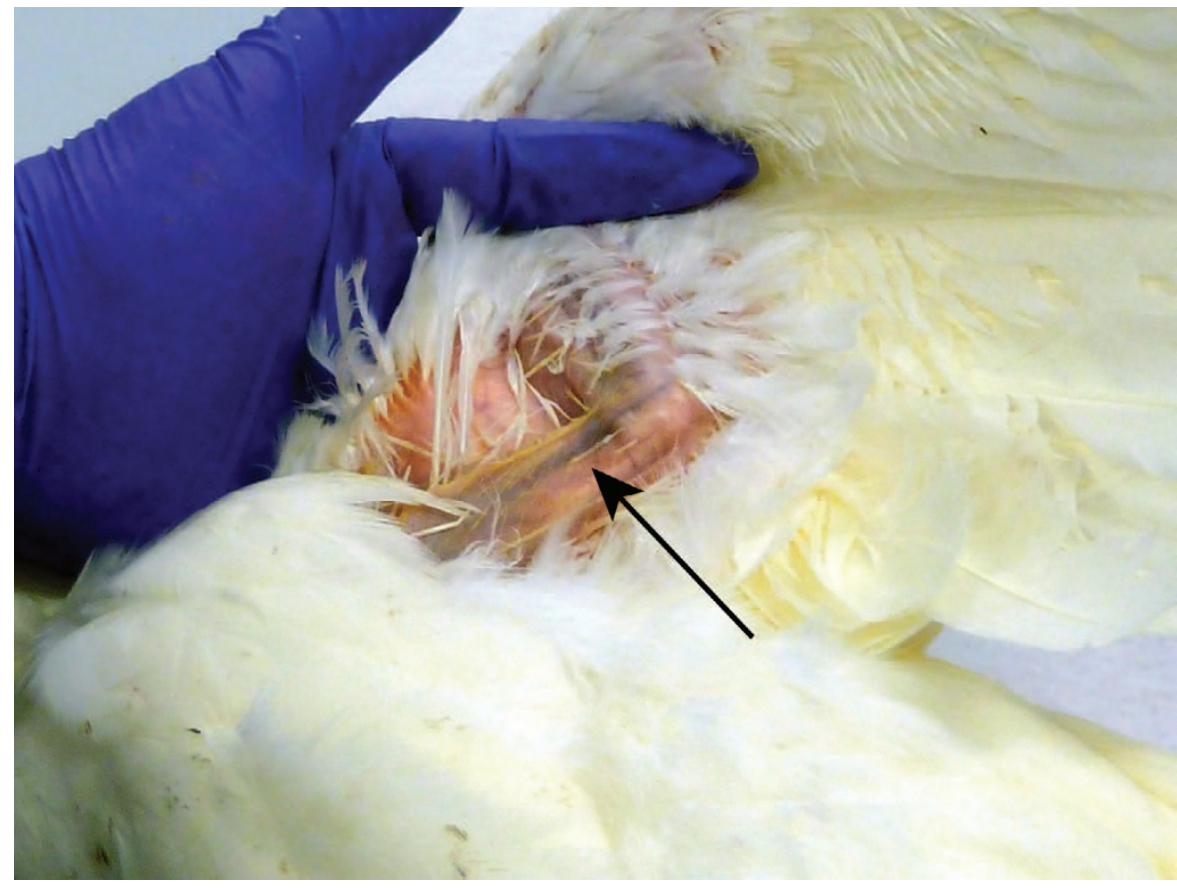

FIGURE 1 | Brachial wing vein location. The chicken is in right lateral recumbency with the left wing extended away from its body. The brachial wing vein (arrow) is located between the biceps and triceps muscle on the underside of the wing.

are easy to handle and adaptable to a wide variety of settings. Chicken red blood cells are nucleated ${ }^{2}$, making DNA extraction from them easier than from mammalian blood cells.

\section{Blood collection recommendations}

Poultry have a relatively small percentage of blood volume by body weight, approximately 6-7.5\% (refs. 3-5). However, they replace blood at a slightly faster rate than do mammals, as avian red blood cells have an average lifespan of 28-35 d (refs. 2, 6) compared with $60 \mathrm{~d}$ in the rat and $40.7 \mathrm{~d}$ in the mouse ${ }^{7}$. No more than $1 \%$ of the body weight equivalent of blood should be taken in a single collection, and the chicken should be allowed at least 14 days to recover before more blood is withdrawn. A single collection of $2 \%$ of the body weight 
may be obtained if warm, isotonic fluids are administered to replace fluid loss.

Positioning and handling of the chicken for phlebotomy are perhaps the most important skills to master. It is preferable for two people to carry out the procedure; one can restrain the bird while the other occludes the vessel and collects the sample. With some practice, however, an unassisted clinician can successfully carry out venipuncture on a conscious chicken. The chicken must be handled gently; it has fragile bones and must be able to move its keel bone (sternum) in order to breathe. Additionally, chickens overheat quickly if they cannot dissipate body heat during restraint. Generally, once a chicken is restrained, it will relax. Placing a cloth over an anxious chicken's head can help to relax the bird. Because chickens are easily startled, sudden movements and loud noises should be avoided when working with chickens.

Blood may be withdrawn from four key sites in poultry: the brachial wing vein, the medial metatarsal vein, the jugular vein and the heart. The volume of blood that can be collected from each site will depend on the weight of the chicken, the skill of the phlebotomist and the speed of blood clotting.

\section{Brachial wing vein}

The brachial wing vein (or cutaneous ulnar vein) is the most common site for blood collection in an adult chicken. This vein can be accessed on the underside of the bird's wing, near the elbow, and feathers can be plucked from this area to aide in visualization. The wing vein runs between the biceps and triceps muscles (Fig. 1) and bifurcates just proximal to the elbow. Blood should be collected at the bifurcation.

For brachial wing venipuncture by two people working together, the bird is placed in lateral recumbency with its feet facing the phlebotomist. The assistant lifts and gently stretches the top wing to expose the vein on the underside, so that the phlebotomist can insert the needle and withdraw the blood. For venipuncture by an unassisted phlebotomist, the bird is placed in lateral recumbency facing the phlebotomist, with its feet pointing toward the phlebotomist's dominant hand and both wings unrestrained. The back legs may be

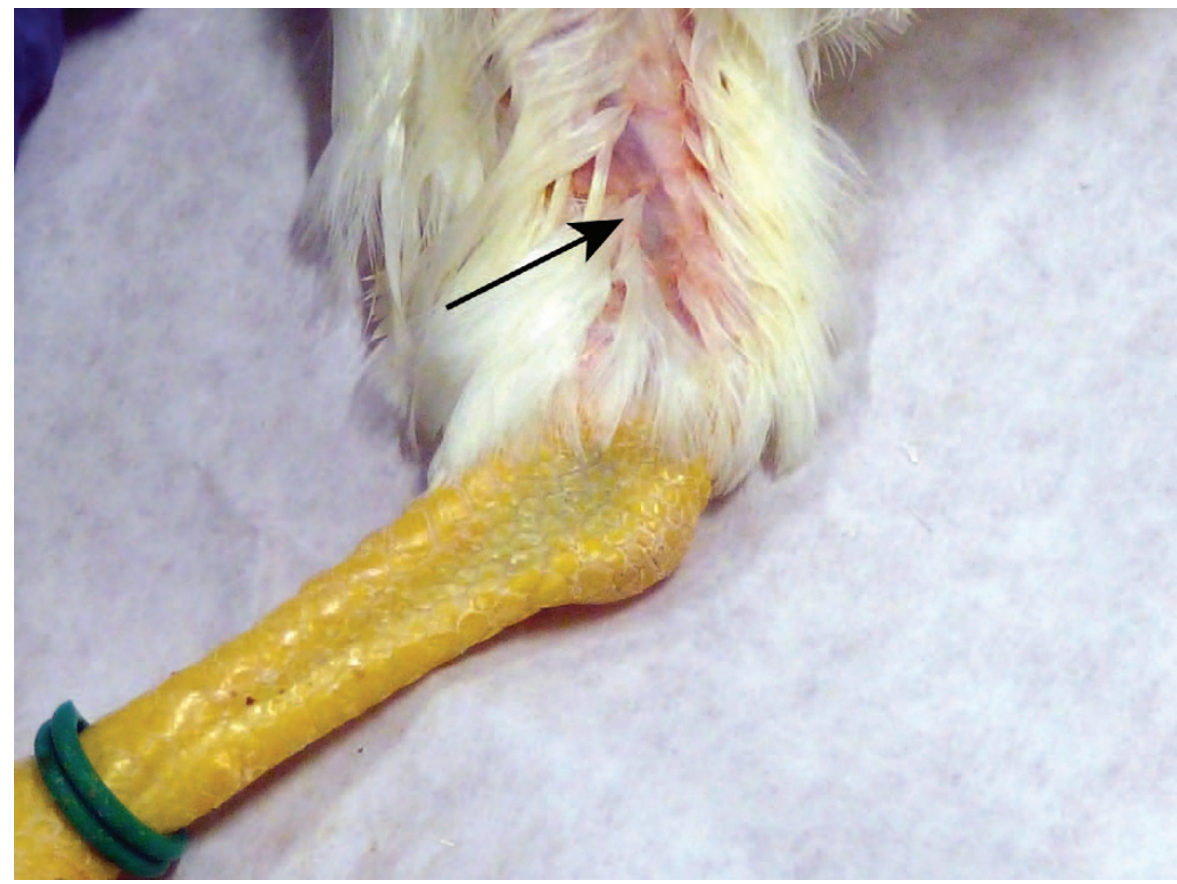

FIGURE 2 | Medial metatarsal vein location. The chicken is in right lateral recumbency with the right leg gently straightened. The medial metatarsal vein is located proximal to the hock joint of the lower leg (arrow).

loosely tied together to enhance restraint. between the second and third finger and The phlebotomist moves his or her non- the top wing between the third and fourth dominant hand down the back of the finger. The thumb on the non-dominant bird's neck, grasping the underlying wing hand can then be used to occlude the

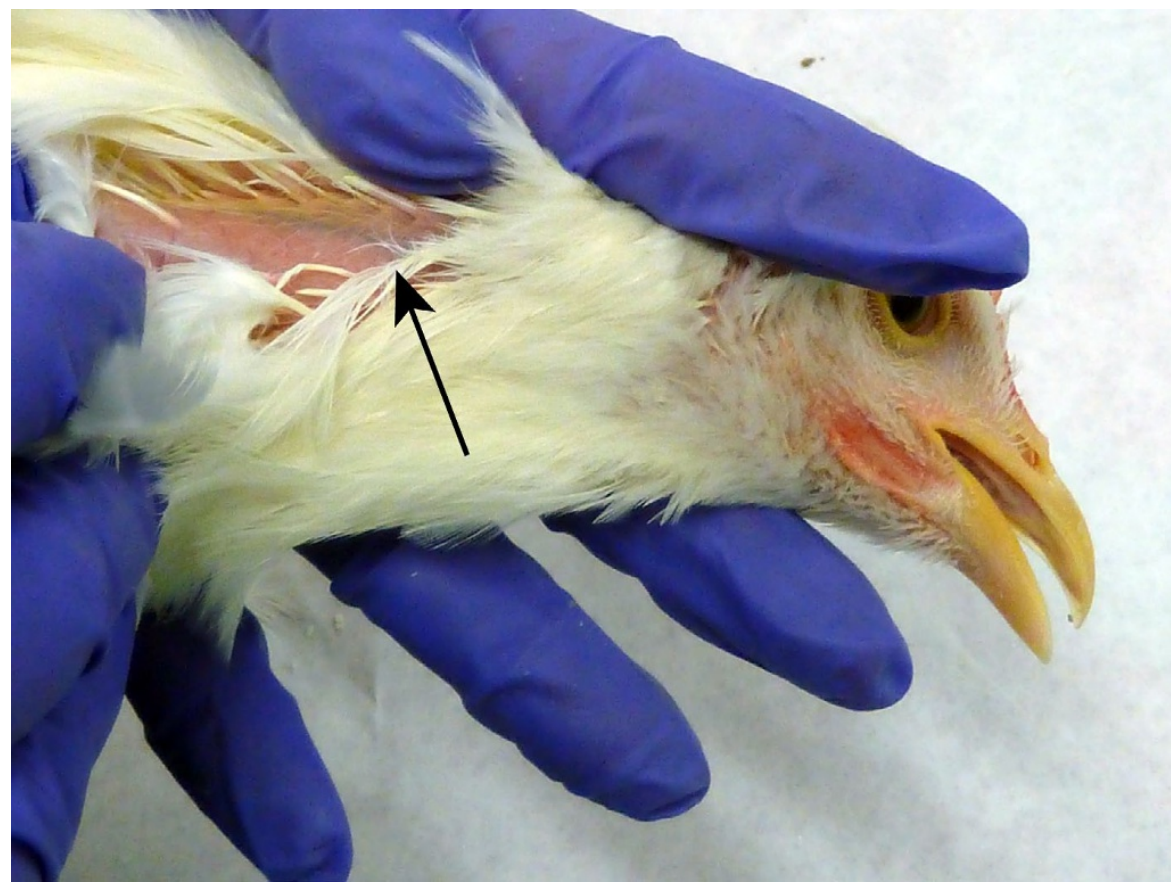

FIGURE 3 | Jugular vein location. The chicken is in left lateral recumbency with the neck gently straightened and lengthened. The jugular vein is located in the featherless tract along the right side of the neck (arrow). 
vein, while the dominant hand is used to insert the needle and withdraw the blood. Alternatively, the phlebotomist can sit and place the bird in dorsal recumbency, using his or her thighs to restrain the bird's body. The phlebotomist then uses his or her non-dominant hand and forearm to lift and stretch the wing on the opposite side. Again, the thumb of the non-dominant hand is used to occlude the vessel, while the dominant hand is used to insert the needle and withdraw the blood.

For sterile blood collection, a 25-gauge, 1 -in-long needle is inserted into the brachial wing vein at a shallow angle (approximately $10-20^{\circ}$ ) with the bevel up. Blood should be withdrawn very slowly. Alternatively, a winged infusion set with a 25 -gauge needle may be used to reduce the risk of hematoma. If a hematoma forms, blood should not be collected from the site since it will likely clot before it can be transferred to a blood collection tube.

For non-sterile blood collection, the area is plucked free of feathers, cleaned and lubricated (e.g., using sterile jelly) to aid the formation of a blood drop. If alcohol is used to clean the area, it should be completely dry before puncture to allow the blood to pool. The brachial wing vein is punctured with a 23-gauge needle or a 4-mm lancet. Blood is collected using a pipettor or a microhematocrit tube.

\section{Medial metatarsal vein}

The medial metatarsal vein in the lower leg (Fig. 2) can be accessed for phlebotomy. This vein is surrounded by muscles, minimizing the risk of hematoma formation, but can be difficult to locate, especially in an adult bird.

For medial metatarsal venipuncture, the chicken is placed in lateral recumbency. The underlying leg is gently straightened, and the feathers are separated along the hock joint. A 25-gauge, 1-in needle is inserted at a $30^{\circ}$ angle with the bevel up. Blood should be withdrawn slowly. Collection from the medial metatarsal vein is often better suited to puncture collection using a 23-gauge, 1 -in needle. For puncture, feathers in this area should be plucked. Application of lubricant may aide in collection of the blood drop by pipettor or microhematocrit tube.

\section{Jugular vein}

The jugular vein is found of the right side of the chicken's neck. This site allows for faster collection of larger volumes of blood, but the vein is very mobile and there is a large amount of subcutaneous space, making the site prone to hematoma formation.

Jugular venipuncture typically requires two people. The bird is placed in left lateral recumbency, with its legs and wings gently restrained. The chicken's head is gently pulled away from the body to straighten and lengthen the neck. A small amount of alcohol is applied to the chicken's neck to enable visualization of the jugular vein in the featherless tract along the right side of the neck (Fig. 3). The phlebotomist occludes the jugular vein with his or her nondominant hand or forefinger at the level of the thoracic inlet. The vein is punctured at a very shallow angle (approximately $10-20^{\circ}$ ) using a 25-gauge, 1-in needle, and blood should be withdrawn slowly. If a hematoma forms, this site should be abandoned. The jugular vein is not an appropriate location for puncture collection.

\section{Heart}

The heart can be punctured for collection of large volumes of blood from anesthetized birds that will not regain consciousness after the blood collection. Typically, the bird is placed in right lateral recumbency and the needle is inserted between the ribs, perpendicular to the skin. The needle length required to reach the heart will depend on the individual bird's size. Chickens are usually exsanguinated using this method.

\section{Post-procedural issues}

After blood collection, compression should be applied to the venipuncture site to assure hemostasis. The chicken should not be returned to its cage or flock until bleeding has stopped. This delay is especially important for young birds, as they may cannibalize bleeding pen mates. Venipuncture techniques are usually minimally stressful; chickens quickly recover and return to normal activities. Hematomas are the most common complication.

\section{ACKNOWLEDGMENTS}

We thank Mr. Greg Eidson for providing access to chickens as photographic subjects and Ms. Allison Watson and Ms. Liz Stich for assistance with photography.

1. Smith, C.P. Animal models in biomedical research: poultry. in Special Reference Briefs Series, 92-17 (National Agricultural Library, Beltsville, MD, 1992). <http://www.nal.usda. gov/awic/pubs/oldbib/srb92-17.htm>

2. Glomski, C.A. \& Pica, A. The Avian Erythrocyte: Its Phylogenic Odyssey (CRC Press, Boca Raton, FL, 2011).

3. Morton, D.B. et al. Removal of blood from laboratory mammals and birds. Lab. Anim. 27, 1-22 (1993).

4. Raj, A.B.M. Stunning and slaughter of poultry. in Poultry Meat Processing and Quality (ed. Mead, G.C.) (CRC Press, Boca Raton, FL, 2004).

5. Medway, W. \& Kare, M.R. Blood and plasma volume, hematocrit, blood specific gravity and serum protein electrophoresis of the chicken. Poult. Sci. 38, 624-631 (1959).

6. Rodnan, G.P., Ebaugh, F.G. Jr., Fox, M.R.S. \& Chambers, D.M. The life span of the red blood cell and the red blood cell volume in the chicken, pigeon and duck as estimated by the use of Na2Cr5104: with observations on red cell turnover rate in the mammal, bird and reptile. Blood 12, 355-366 (1957).

7. Van Putten, L.M. \& Croon, F. The life span of red cells in the rat and the mouse as determined by labeling with DFP32 in vivo. Blood 13, 789-794 (1958). 